\title{
Breast screening remains a controversial issue
}

\author{
A L Harris ${ }^{\star}, 1$ \\ ${ }^{1}$ The Weatherall Institute of Molecular Medicine, University of Oxford, John Raddliffe Hospital, Oxford OX3 9DS, UK
}

The Marmot Report, published in full in this issue of British Journal of Cancer (Marmot et al, 2013), examines in detail the value of breast screening. Although a shortened version has been published previously (Independent UK Panel on Breast Cancer Screening, 2012), the Editorial Board felt that this is such an important issue in terms of resources, controversy and cost-benefit analysis, as well as potential patient benefit, that the whole report should be published in full here.

We have invited editorial comment from leaders in the field, who have previously contributed to reviewing the value of breast screening, to give their position and interpretation of the report. It is clear that as randomised breast screening was developed, there have been improvements in screening technologies, major improvements in survival with treatment used for adjuvant therapy and also recurrent disease. Although there has usually been a delay of at least a year in the United Kingdom taking up new drug treatment in the adjuvant situation, all current approved drugs are available in the UK, including aromatase inhibitors, herceptin and the taxanes, and it is now possible also to access drugs for metastatic disease on a routine basis for example, exemestane and evorlimus, eribulin and lapatinib. However, as successive generations of chemotherapy and anti-hormone therapy have improved the outcome, it becomes increasingly difficult to assess the value of breast screening compared with therapy. As Professor Baum (2013) points out, probably this issue can never be resolved without new randomised studies using optimised screening approaches and prediction algorithms $v s$ optimised therapy for the primary disease - the better the treatment and outcome of the primary symptomatic disease, the less the benefit of screening will be.

It is important to consider how the initiation of the screening programme introduced many very important organisational changes in the management of breast cancer, including optimised diagnostic services in pathology and radiology with national and local audits and quality developments. There was further development of multidisciplinary teams including all those delivering the treatment, such as surgical, radiation and medical oncologists and specialist nurses, with regular team meetings to internally review and ensure national standards were kept and timelines for patient management were maintained.

It is unlikely that this would have happened without the funding available to set up the screening programme and the pathology and radiology required, because this is essential infrastructure on which everything else is built. So, one needs to consider that the optimised workload patterns, integrated management teams, national quality standards and early adoption of international protocols have all contributed substantially to the improvement of outcome for breast cancer in the United Kingdom.

We welcome correspondence on the report.

\section{REFERENCES}

Baum M (2013) The Marmot report: accepting the poisoned chalice. Br J Cancer 108(11): 2198-2199.

Independent UK Panel on Breast Cancer Screening (2012) The benefits and harms of breast cancer screening: an independent review. The Lancet 380(9855): 778-786.

Marmot G, Altman DG, Cameron DA, Dewar JA, Thompson SG, Wilcox M. The Independent UK Panel on Breast Cancer Screening (2013) The benefits and harms of breast cancer screening: an independent review. Br J Cancer 108(11): $2205-2240$.

(1) (2) (2) This work is licensed under the Creative Commons Attribution-NonCommercial-Share Alike 3.0 Unported License. To view a copy of this license, visit http://creativecommons. org/licenses/by-nc-sa/3.0/ 